\title{
Experimental investigation of torsional vibration isolation using Magneto Rheological Elastomer
}

\author{
Praveen Shenoy $K^{1^{*}}$, Abhishek Kumar Singh ${ }^{1}$, Sai Aditya Raman $K^{1}$, and $K . V$. \\ Gangadharan ${ }^{1}$ \\ ${ }^{1}$ Centre for System Design, National Institute of Technology Karnataka, Surathkal, Mangalore- \\ 575025, Karnataka, India
}

\begin{abstract}
Rotating systems suffer from lateral and torsional vibrations which have detrimental effect on the roto-dynamic performance. Many available technologies such as vibration isolators and vibration absorbers deal with the torsional vibrations to a certain extent, however passive isolators and absorbers find less application when the input conditions are dynamic. The present work discusses use of a smart material called as Magneto Rheological Elastomer (MRE), whose properties can be changed based on magnetic field input, as a potential isolator for torsional vibrations under dynamic loading conditions. Carbonyl Iron Particles (CIP) of average size $5 \mu \mathrm{m}$ were mixed with RTV Silicone rubber to form the MRE. The effect of magnetic field on the system parameters was comprehended under impulse loading conditions using a custom built in-house system. Series arrangement of accelerometers were used to differentiate between the torsional and the bending modes of vibration of the system. Impact hammer tests were carried out on the torsional system to study its response, in the presence and absence of magnetic field. The tests revealed a shift in torsional frequency in the presence of magnetic field which elucidates the ability of MRE to work as a potential vibration isolator for torsional systems.
\end{abstract}

\section{Keywords}

Torsional Vibrations, Isolation, Impact hammer tests, Magneto Rheological elastomer

\section{Introduction}

Torsional vibrations have been a subject of study due to its role in causing catastrophic failure in many of the rotating members. All systems subjected to rotation involve torsional oscillations during start up or shut down of prime mover. During this, the system may pass through the torsional natural frequencies of the system. Since the time for which the system dwells in the frequency is less, the possibility for a failure is minimum. However prolonged modes of operation may lead to an imminent failure of the system. Hence an in-depth study of reduction of torsional vibrations in the rotating systems has been one of the prominent aspects in the design of rotating systems 12.

\footnotetext{
${ }^{*}$ Corresponding Author: praveen.me14f12@,nitk.edu.in
} 
Reducing vibration in systems has been carried out in various ways. Vibration absorbers and vibration isolation concepts have been used effectively in arresting translatory as well as rotational vibrations of systems. Absorbers employ the fact that adding a secondary mass to the primary system of interest reduces the amplitude of vibration on the primary system. Isolators on the other hand rely heavily on the system's inherent properties (mainly stiffness and damping) to reduce the amplitudes of critical oscillations. The present paper focusses on the latter.

Vibration isolation has been efficiently carried out in systems in various ways. It involves changing the stiffness or the damping of the system between the source of disturbance (Base) and the source of interest (Mass/Inertia). Based on the controllability, isolators are divided into "Passive, Active and Semi-active" systems. Passive isolation systems have been successfully implemented in many applications for it is economical and very easy to be built into. However it fails to work for systems with varying properties of stiffness and damping. Active isolators resolve this issue as they have a feedback supplied to the actuation unit. Due to their bulky nature and sophistication, they have been popular only within a limited branch of dynamics. Semi-active isolators bridge the gap between the two. They incorporate the simple yet effective characteristic of the passive systems and the ability to change the properties of the systems as that of the active systems.

One aspect that sets the semi active and the active vibration systems apart is the ability of the material to change its inherent property. Such materials are called Smart Materials. Many smart materials such as piezo materials, shape memory alloys, magnetostrictive materials and newer forms of composites have been used in the effective mitigation of vibration amplitudes. One such material is called Magneto Rheological Elastomer also known as MRE. MRE is the solid form in the family of smart materials called as Magneto Rheological materials. Its fluid analogue, the Magneto Rheological Fluids have been used as fluid dampers in suspension systems for their excellent damping capabilities. MRE on the other hand have been used for vibration isolation under dynamic loading conditions. It consists of a viscoelastic matrix material in which the iron particles are imbibed either randomly (Isotropic) or along the direction of induced magnetic field during its curing process (Anisotropic). The elastomer upon curing forms into a solid form and on application of magnetic field, the iron particles arrange themselves as chains in the direction of the applied field. Due to this, the material undergoes changes in its properties. This has been used as isolators in systems with varying ranges of natural frequency making it to be efficiently used in semi-active and active vibration isolation systems.

MRE have been extensively studied for its isolation properties. Since the initial work carried out by Ginder et al. 3, numerous papers deal with MRE's isolation capabilities. However the isolation capabilities of MRE have been studied in the translatory modes of vibration. Liao et al. 4 worked on real time tunable vibration isolators with multiple layers of MRE. Jeong et al. 5 carried out designing a MRE elastomer differential mount which was used in between the engine and the car body. MRE has also been studied in seismic vibration isolation. Jung et al. 6 carried out base vibration tests on MRE with various forms of input being given to the structure. Behrooz et al. 78 studied the variable stiffness and damping in base isolation. An increase of $57 \%$ in the shear performance was observed. It has to be noted that the frequency of operation for which the isolation studies have found to be effective is in the lower range (i.e., in the range of 1 to $100 \mathrm{~Hz}$ ). Hegde et al. 9 examined the effect of particle size on the dynamic damping of the MRE. Transmissibility ratio for two particle sizes were compared along with the loss factor. It was found that the smaller particle sized MRE showed better improvement on loss factor compared to pure silicone due to agglomeration of the particles.

Research has been carried out in using MRE as a potential semi-active vibration absorber in powertrain systems. Hoang et al. 11 carried out numerical simulations on a vibration 
absorber. An adaptive tunable vibration absorber (ATVA) based on MRE was designed and developed. The effectiveness of ATVA was evaluated in terms of shift in the resonant frequencies was numerically investigated on powertrain systems.

Experimental modal analysis provides an excellent way to determine many of the modal parameters. The modal parameters are determined using the Frequency Response Functions (FRFs) which is the ratio of the output response to the input force which is given to the system. The input force may be given in-terms of modal shakers or using an impact hammer which gives impulse input. The present paper deals with understanding the torsional frequency variation under the influence of magnetic field using FRF response.

\section{Methodology}

\subsection{MRE Preparation}

An RTV based silicone material is used as the matrix with Carbonyl Iron Particles (CIP) of an average diameter of $5 \mu \mathrm{m}$ mixed into it. The volume fraction of the matrix to the CIP is 73:27. The details of the constituent materials are as shown in Table 1:

Table 1: MRE composition

\begin{tabular}{|c|c|c|c|}
\hline SI No & Constituents & Make & Density $\left(\mathbf{k g}_{\mathbf{m}^{\mathbf{3}}}\right)$ \\
\hline 1 & RTV Based Silicone rubber & Dow Corning & 884.57 \\
\hline 2 & Carbonyl Iron Particles & BASF, Type CN & 7850 \\
\hline
\end{tabular}

The procedure involves mixing the RTV silicone with the CIP in the mentioned concentration. The mixture is stirred continuously till the constituent materials get mixed thoroughly. This mixture is then poured into the mould and is degassed until the air bubbles are removed. This is subjected to curing for a period of 20 hours under constant pressure at room temperature.

\subsection{Experimental Setup}

The schematic of the experimental setup is as shown in Fig. 1. It consists of two Aluminum shafts connected via the MRE coupler and one Mild Steel disk mounted on each of the shafts at the free end. To measure the torque, two HBM T22, $10 \mathrm{Nm}$ Torque transducers (TT) was used on either side of the isolator. The Torque transducers and the bearings were firmly supported on the rigid base. Further an YMC Piezotronics Inc. single axis accelerometer was used to measure the frequency response of the base. In order to extract the bending and the torsional modes of the system separately, four triaxial accelerometers (Measurement Specialities Inc), two on either disks were used. A PCB Piezotronics impact hammer was used to provide the impulse force. To induce magnetic field, permanent magnets (type N52, Neodymium magnets) were used. A Lake Shore, (Model 410) Gaussmeter was used to measure the generated magnetic field. The data obtained from the sensors was read through NI 9234, a National Instrument Data Acquisition unit into a NI PXIe-1082 which was further read through LabVIEW 2014 (Fig. 2)

Prior to finding out the bending and the torsional modes of vibrations, a modal analysis was carried out on the rigid base member (Fig. 3) using Roving hammer test. This was done to analyse the predominant frequencies of the base which could have a likely effect on the system's natural frequency. This is proceeded with finding the bending and the torsional modes of vibrations using the Serial Accelerometer Arrangement (SAA) and the Parallel 
Accelerometer Arrangement (PAA) of the two tri-axial accelerometers 12 as shown in Fig. 4. The frequencies corresponding to the peak amplitudes from the response graphs determine the torsional and the bending modes of vibrations. Once the torsional modes was found, the effect of field on the torsional natural frequencies and the amplitude was studied. To impart torsional oscillations on the system, an extension is attached on the inertial element (disc), and an impact is given on end of the extension as shown in Fig. 2. Similar approach has been carried out to impart torsional oscillations on systems 1314 . The frequency resolution is 0.1 $\mathrm{Hz}$ with the number of averages considered is 10 .

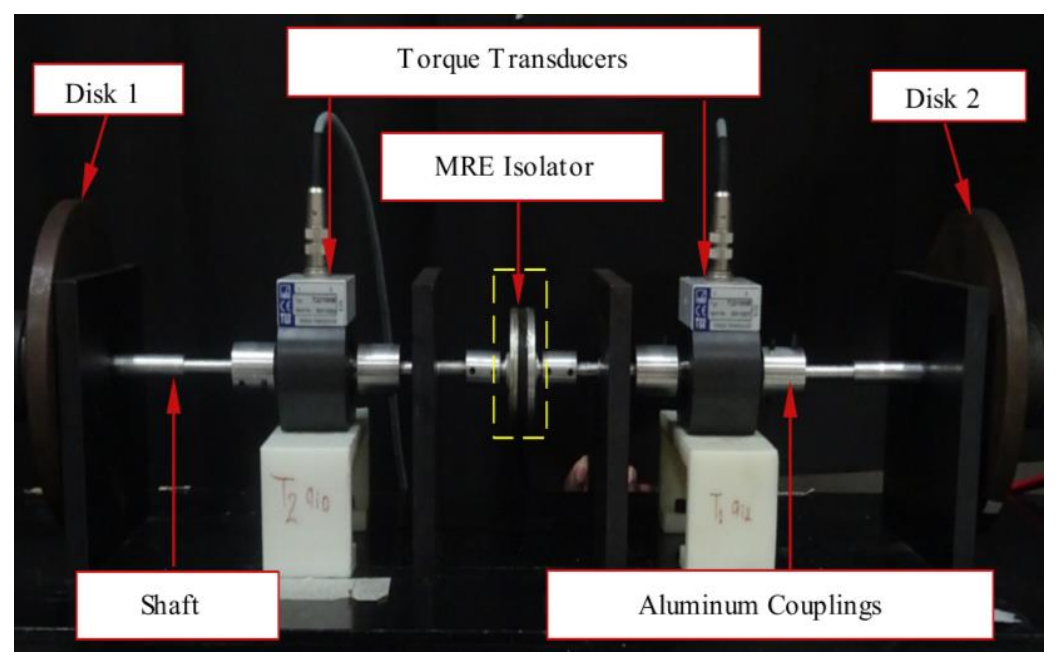

Fig. 1. Torsional 2 DoF system

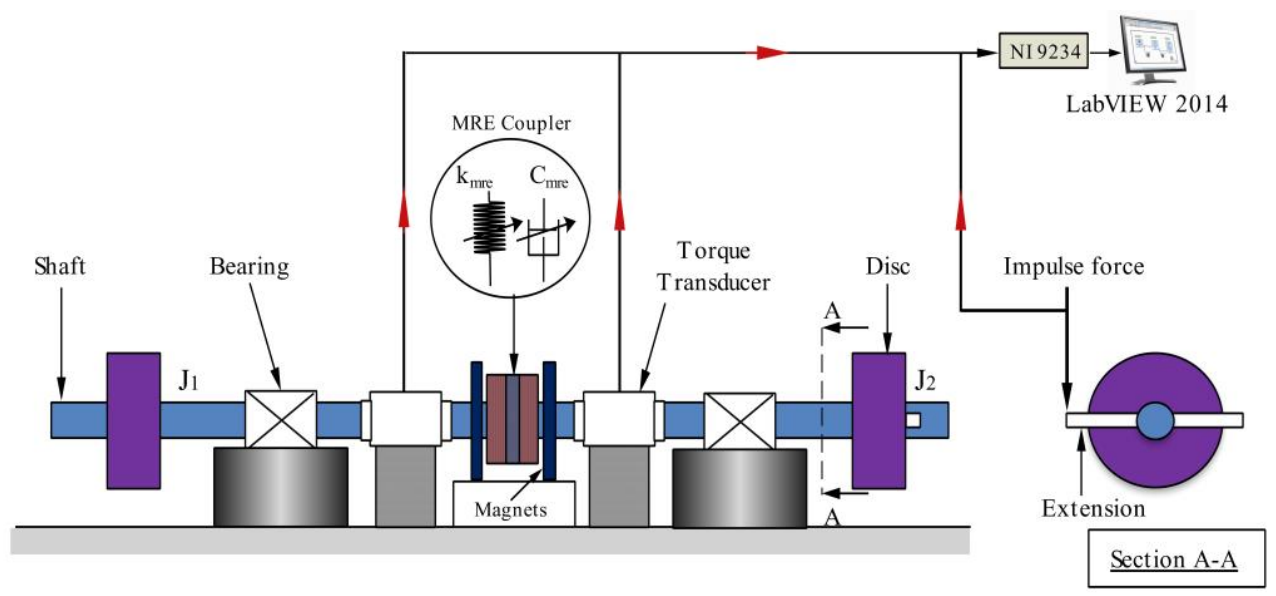

Fig. 2. Schematic representation of experimental setup 


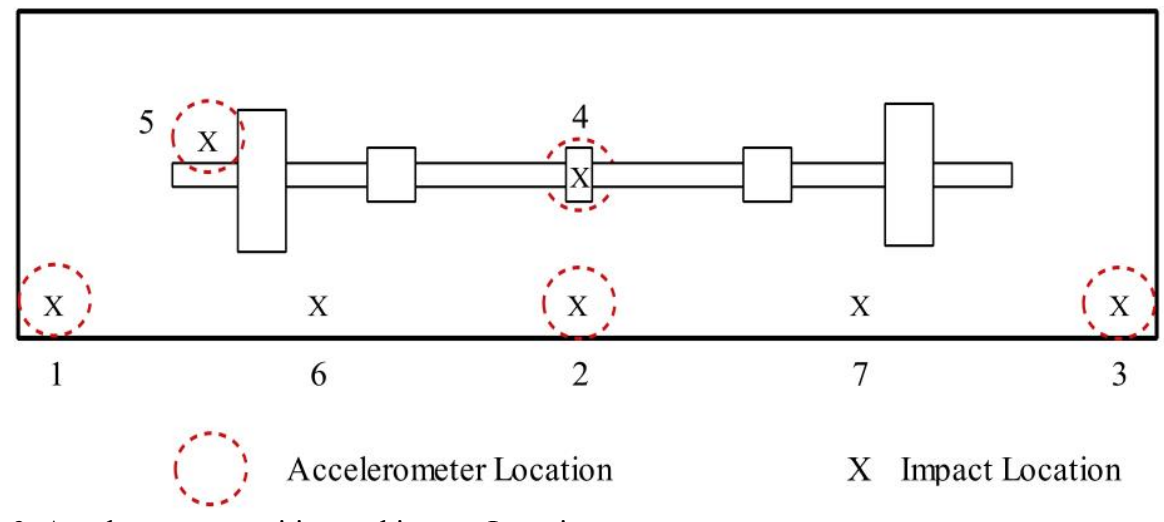

Fig. 3. Accelerometer position and impact Locations

Kuang et al. 12 developed a two accelerometer system to evaluate the bending and torsional natural frequencies of a shaft-rotor system. Two accelerometers in parallel and serial combinations have been used and the corresponding natural frequencies in bending and torsion were calculated. Similar approach is applied in the present set-up to obtain the torsional natural frequencies of the system.

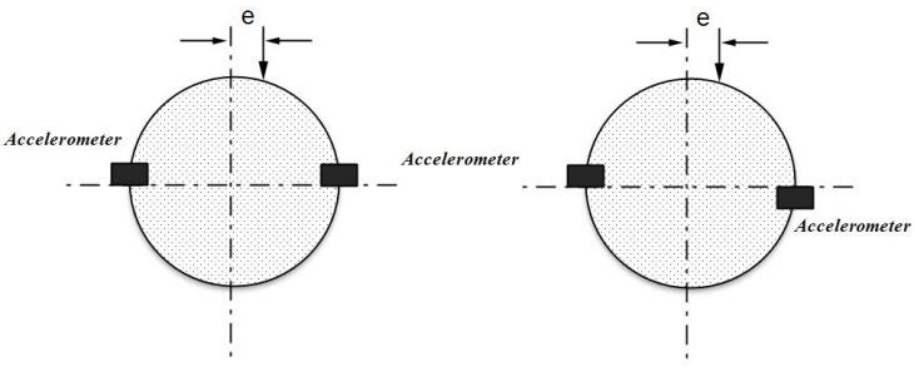

Fig. 4. Parallel and Serial arrangements of Accelerometers

For the serial and parallel arrangement of the accelerometers, the equations 12 of the harmonic response is given as,

wherein:

$$
\begin{gathered}
P_{i j}(\omega)=2\left\{\sum_{r=1}^{n} \frac{\emptyset_{r i} \emptyset_{r j}}{\omega_{r}^{2}\left[1-\left(\frac{\omega}{\omega_{r}}\right)^{2}\right]}\right\} F_{j}(\omega) \\
S_{i j}(\omega)=2 r\left\{\sum_{r=1}^{m} \frac{\theta_{r i} \theta_{r j}}{\Omega_{r}^{2}\left[1-\left(\frac{\omega}{\Omega_{r}}\right)^{2}\right]}\right\} T_{j}(\omega)
\end{gathered}
$$

$P_{i j}$ and $S_{i j}$ are the harmonic response in PAA and SAA measurement $\emptyset_{r i} \emptyset_{r j}$ and $\theta_{r i} \theta_{r j}$ Are the linear displacement and the angular displacement terms $\omega_{r}$ and $\Omega_{r}$ are the bending mode and torsional mode natural frequencies $F_{j}$ and $T_{j}$ are the harmonic force and harmonic torque applied on the disks as,

For a single degree of freedom system the harmonic signal in torsional mode is given 


$$
S(\omega)=2 r\left\{\sum_{r=1}^{m} \frac{\theta^{2}}{\Omega_{r}{ }^{2}\left[1-\left(\frac{\omega}{\Omega_{r}}\right)^{2}\right]}\right\} T_{j}(\omega)
$$

From the above equation, the FRF function for the torsional modes of frequencies can be obtained as,

$$
F R F=\frac{S(\omega)}{T_{j}(\omega)}=2 r\left\{\sum_{r=1}^{m} \frac{\theta^{2}}{\Omega_{r}^{2}\left[1-\left(\frac{\omega}{\Omega_{r}}\right)^{2}\right]}\right\}
$$

\section{Results and discussion}

\subsection{Impact test results on the Torsional vibration system base}

As discussed in the previous section, the primary aim was to identify if any of the natural frequencies of the base structure are close to torsional natural frequencies of interest. Table 2 indicates the lowest frequencies at which peaks were observed in inertance FRFs obtained at various positions (1-5) when impact was given at locations (i) to (vii) shown in Fig. 3.

Table 2. Lowest identified critical frequencies based on FRF curves for seven input and five response positions. (All frequencies are in $\mathrm{Hz}$ )

\begin{tabular}{|c|c|c|c|c|c|}
\hline & \multicolumn{5}{|c|}{ Accelerometer positions } \\
\hline Impact position & $\mathbf{1}$ & $\mathbf{2}$ & $\mathbf{3}$ & $\mathbf{4}$ & $\mathbf{5}$ \\
\hline (i) & 145 & 600 & 600 & 535 & 820 \\
\hline (ii) & 270 & 550 & 820 & 550 & 315 \\
\hline (iii) & 260 & 300 & 580 & 555 & 820 \\
\hline (iv) & 250 & 290 & 600 & 600 & 820 \\
\hline (v) & 115 & 110 & 110 & 350 & 120 \\
\hline (vi) & 280 & 100 & 280 & 100 & 500 \\
\hline (vii) & 270 & 320 & 475 & 560 & 480 \\
\hline
\end{tabular}

From the table it is seen that the least frequency component obtained is $100 \mathrm{~Hz}$ and 110 $\mathrm{Hz}$ with the mountings on the base. This implies that the base frequency components do not affect the torsional natural frequencies which are expected to be within $50 \mathrm{~Hz}$ corresponding to the working torsional frequency range of many of the rotating systems. It is seen that the addition of the mountings increases the natural frequency of the base to $115 \mathrm{~Hz}$. This is due to the fact that the mountings result in increase of the stiffness of the base structure.

\subsection{Determination of torsional and bending modes using accelerometer pair measurements}

Fig. 5 and Fig. 6 show the torsional and bending modes of the disk-shaft system with the serial and parallel arrangement of the accelerometers. As seen, the serial arrangement of the accelerometers reveal the torsional mode of vibration which occurs at approximately $13.5 \mathrm{~Hz}$ $(780 \mathrm{rpm})$ which is the speed of interest for many of the torsional systems. 


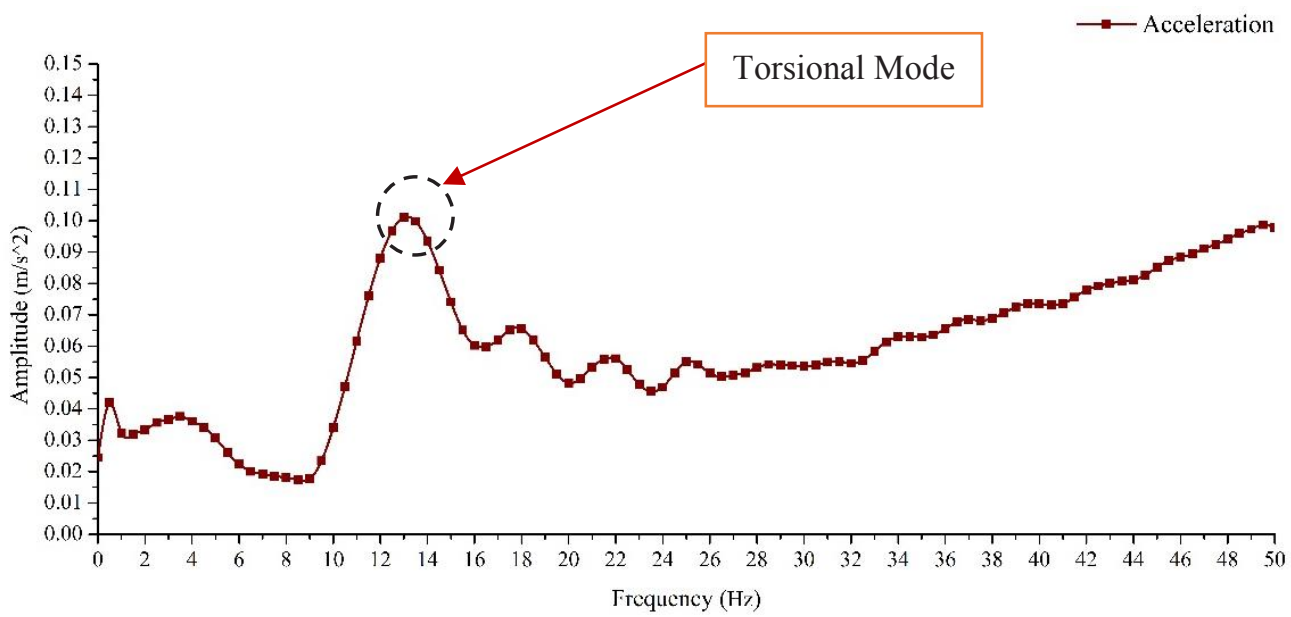

Fig. 5. Accelerometers reading for serial arrangement

In the parallel arrangement of accelerometers, bending mode of system is enhanced while torsional mode is cancelled due to phase lag in accelerometers. It is seen that the bending mode occurs at approximately $42 \mathrm{~Hz}$ which is found to be away from the torsional mode of vibration. From this it is seen that under the frequency range of interest (i.e., $1 \mathrm{~Hz}$ to $50 \mathrm{~Hz}$ ), there is only the torsional natural frequency seen and with this, the effect of magnetic field on the stiffness is investigated.

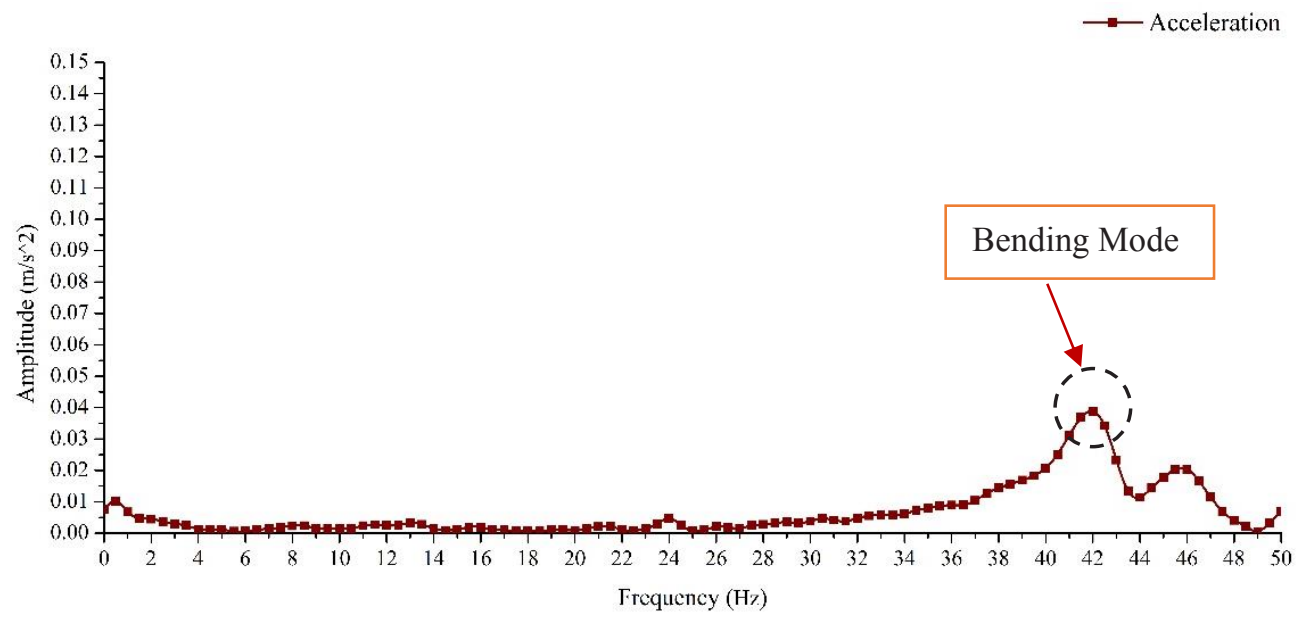

Fig. 6. Accelerometers reading for parallel arrangement

\subsection{Variation in torsional natural frequency under the influence of magnetic field}

The system is subjected to impact hammer tests and the response is analysed. Two torque transducers, one measuring the input torque and the other measuring the output (both with respect to the input impact force), is used to obtain the FRF. Fig. 7 and Fig. 8 show the response of the system in the absence and presence of magnetic field for the input (Torque transducer-1) and the output (Torque transducer-2) transducers. 
The FRF graph for TT-1 is as shown in Fig. 7. The initial DC component is neglected. From the graph it is seen that under 0T (no field condition), the FRF peaks up at approximately $13.5 \mathrm{~Hz}$, which represents the torsional natural frequency of the system. However under the influence of magnetic field (i.e. 0.15T), it is seen that the FRF shows a frequency shift to $14.5 \mathrm{~Hz}$ along with a reduction in amplitude, $\mathrm{H}(\omega)$. Similar case is seen in Fig. 8 with the torque transducer-2.

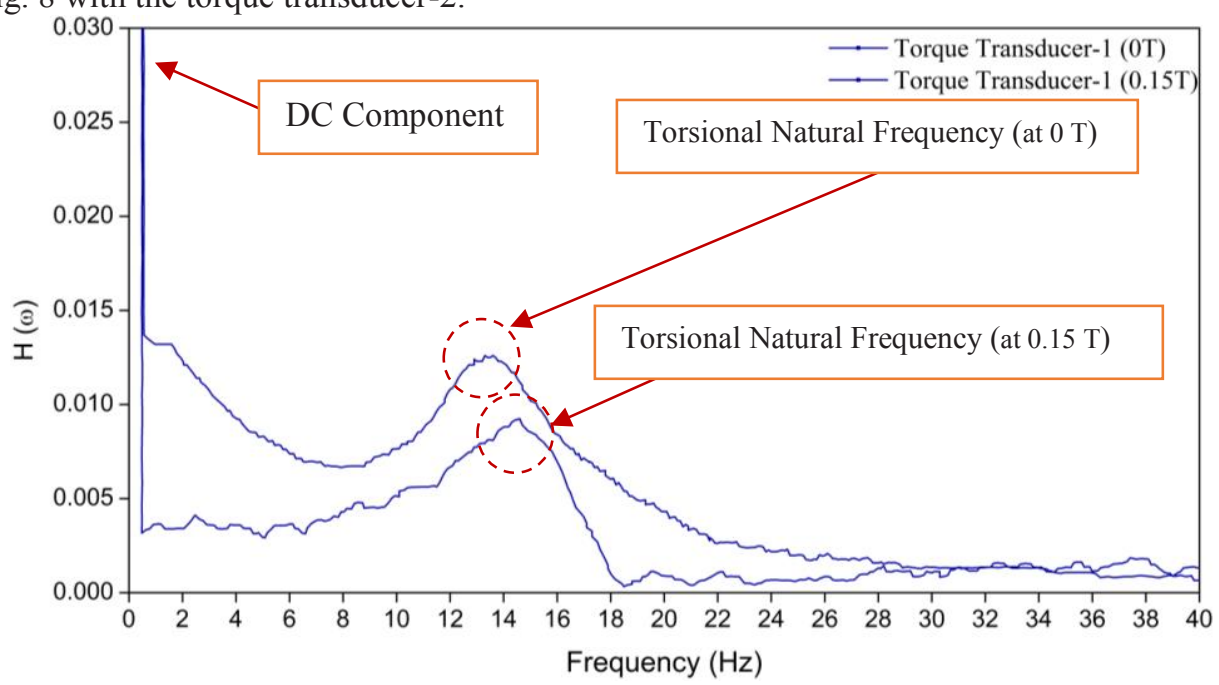

Fig. 7. FRF for Torque transducer-1

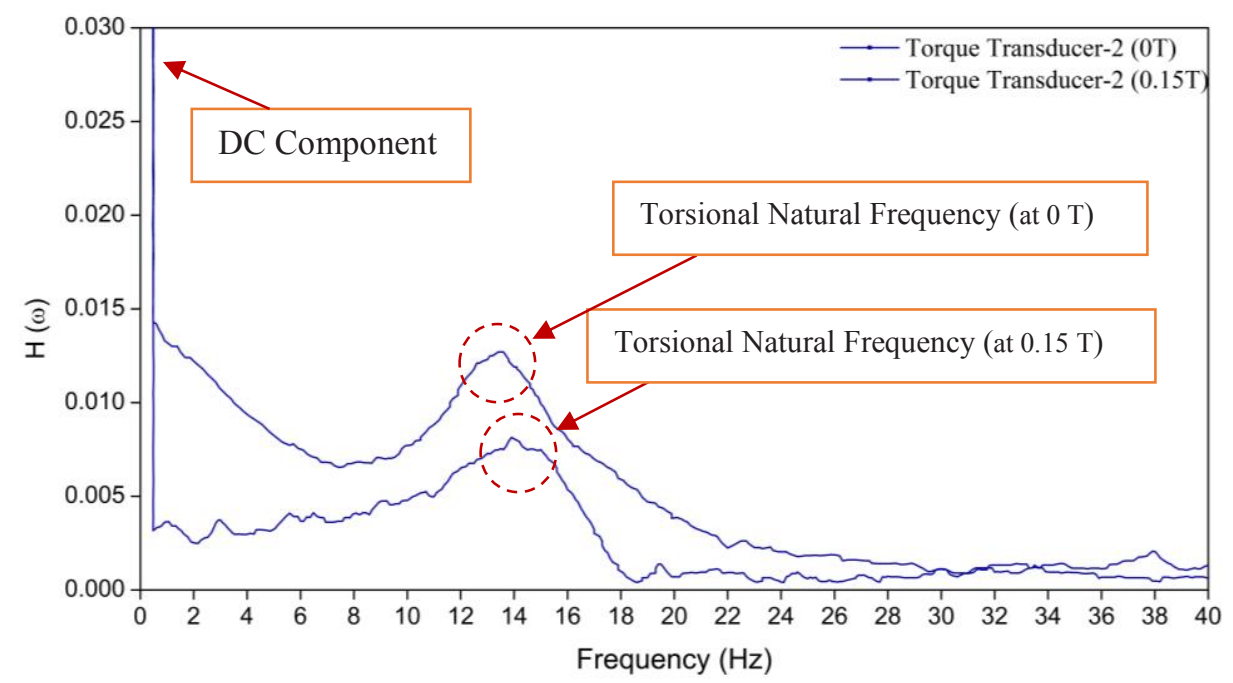

Fig. 8. FRF for Torque transducer-2

From the graphs it is seen that the absolute shift in the frequency is approximately $7 \%$ when the field is increased to $0.15 \mathrm{~T}$. The shift in the torsional natural frequency is attributed to the influence of magnetic field on the properties of MRE. Under the influence of the magnetic field, there is an enhancement of dynamic stiffness of the elastomer which results in increase of the natural frequency of the MRE.

This enhancement in stiffness can be attributed to the compressive forces generated on the matrix material due to CIP (Fig. 9). For simplicity a unit cell of the CIP-Matrix is considered 1516 17. Due to the magnetic field lines, the CIPs get aligned along the magnetic 
field and this in-turn forms a compressive force on the intermediate matrix which in turn increase the stiffness of the unit cell of the CIP-Matrix. When the bulk of the elastomer is considered, this results in the overall stiffness increase of the material.
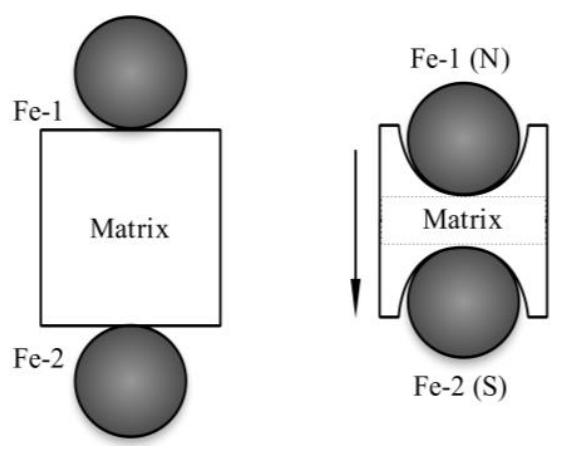

Fig. 9. Unit cell representation of the MRE under non-magnetized and magnetized condition

It is also seen from the FRF plots that there is a reduction in amplitudes at the resonant frequency in the $0 \mathrm{~T}$ and $0.15 \mathrm{~T}$ curves. Table 3 shows the comparison between the FRF amplitudes between the two conditions.

$$
\text { Absolute Reduction }=\frac{\text { Amplitude }(0 \mathrm{~T})-\text { Amplitude }(0.15 \mathrm{~T})}{\text { Amplitude }(0.15 \mathrm{~T})} \times 100
$$

Table 3. FRF amplitude comparison under $0 \mathrm{~T}$ and $0.15 \mathrm{~T}$

\begin{tabular}{|c|c|c|}
\hline Torque Transducers & Amplitude (at 0 T frequency) & $\begin{array}{c}\text { Absolute Reduction } \\
(\mathbf{\%})\end{array}$ \\
\hline TT 1 (Input Side -0 T) & 0.0126 & \multirow{2}{*}{35.6} \\
\hline TT 1 (Input Side $-0.15 \mathrm{~T})$ & 0.00812 & \multirow{2}{*}{40.6} \\
\hline TT 2 (Input Side -0 T) & 0.0127 & \\
\hline TT 2 (Input Side $-0.15 \mathrm{~T})$ & 0.00754 & \\
\hline
\end{tabular}

\section{Conclusions}

The isolation capabilities of Magneto Rheological Elastomer has been investigated experimentally using the impact hammer technique. It was seen that in the presence of field, the natural frequency of the system shifted from $13.5 \mathrm{~Hz}$ to $14.5 \mathrm{~Hz}$. This was ascribed to the increase in the stiffness of the MRE in the presence of field. The well-established di-pole mechanism was used to explain the effect. Also there was a reduction in the amplitude of 0T field, in the presence of field. This proved that the MRE, under torsional shear, worked as an effective isolator.

The authors acknowledge the support from SOLVE: The Virtual Lab @ NITK (Grant number: No.F.16-35/2009-DL, Ministry of Human Resources Development) and experimental facility provided by Centre for System Design (CSD): A Centre of excellence (http://csd.nitk.ac.in/) at National Institute of Technology Karnataka, India 


\section{References}

1. Szenasi, Fred R., and J. C. Wachel. "Analysis of torsional vibrations in rotating machinery." Proceedings of the Turbomachinery Symposium. Vol. 22. 1993.

2. Vance, J. M., and R. S. French. "Measurement of torsional vibration in rotating machinery." Journal of Mechanisms, Transmissions, and Automation in Design 108.4 (1986): 565-577

3. Ginder J M, Nichols M E, Elie L D and Tardiff J L 1999 Magnetorheological elastomers: properties and applications Smart Structures and Materials 1999: Smart Materials Technologies, 131-138, 1999

4. Liao G J, Gong X L, Xuan S H, Kang C J and Zong L H 2012 Development of a real-time tunable stiffness and damping vibration isolator based on magnetorheological elastomer J. Intell. Mater. Syst. Struct.23 25-33

5. Jeong U C, Yoon J H, Yang I H, Jeong J E, Kim J S, Chung K H and Oh J E 2013 Magnetorheological elastomer with stiffness-variable characteristics based on induced current applied to differential mount of vehicles Smart Mater. Struct. 22 115007

6. Jung H-J, Eem S-Hy, Jang D-D and Koo J-H 2011 Seismic Performance analysis of a smart base isolation system considering dynamics of MR elastomer J. Intell. Mater. Syst. Struct. 22 1439-50

7. Behrooz M, Wang X and Gordaninejad F 2014 Performance of a new magnetorheological elastomer isolation system Smart Mater. Struct. 23045014

8. Behrooz M, Wang X and Gordaninejad F 2014 Modeling of a new semiactive/passive magnetorheological elastomer isolator Smart Mater. Struct. 23 045013

9. Hegde, Sriharsha, Umanath R. Poojary, and K. V. Gangadharan. "Experimental investigation of effect of ingredient particle size on dynamic damping of RTV Silicone base Magnetorheological elastomers." Procedia Materials Science 5 (2014): 2301-2309

10. Hoang, N., et al. "Development of a torsional dynamic absorber using a magnetorheological elastomer for vibration reduction of a powertrain test rig." Journal of Intelligent Material Systems and Structures 24.16 (2013): 20362044

11. Hoang, N., N. Zhang, and H. Du. "A dual adaptive tunable vibration absorber using MREs for vehicle powertrain vibration control." SPIE, 2010

12. Kuang, Jao-Hwa, and Jing-Fung Lin. "Accelerometer Pair Measurements for Shaft Dynamic Parameters Analysis." PROCEEDINGS-SPIE THE INTERNATIONAL SOCIETY FOR OPTICAL ENGINEERING. SPIE INTERNATIONAL SOCIETY FOR OPTICAL, 1994

13. Yesilyurt, Isa, and Habibe Gursoy. "Estimation of elastic and modal parameters in composites using vibration analysis." Journal of Vibration and Control 21.3 (2015): 509-524

14. Alwan, V., et al. "Dynamic analysis of shafts of composite materials." Journal of Reinforced Plastics and Composites 29.22 (2010): 3364-3379

15. Poojary, Umanath R., Sriharsha Hegde, and K. V. Gangadharan. "Dynamic blocked transfer stiffness method of characterizing the magnetic field and frequency dependent dynamic viscoelastic properties of MRE." Korea-Australia Rheology Journal 28.4 (2016): 301-313

16. Jolly, Mark R., J. David Carlson, and Beth C. Munoz. "A model of the behaviour of magnetorheological materials." Smart Materials and Structures5.5 (1996): 607 
17. Poojary, Umanath R., Sriharsha Hegde, and K. V. Gangadharan. "Dynamic deformation-dependent magnetic field-induced force transmissibility characteristics of magnetorheological elastomer." Journal of Intelligent Material Systems and Structures (2016): 1045389X16672730 